\title{
PTEN expression and methylation status in oral squamous cell carcinoma
}

\author{
YOSHINORI KURASAWA ${ }^{1}$, MASASHI SHIIBA ${ }^{1}$, MEGUMI NAKAMURA ${ }^{1}$, \\ KAZUAKI FUSHIMI ${ }^{1}$, TAKASHI ISHIGAMI ${ }^{1}$, HIROKI BUKAWA ${ }^{2}$, \\ HIDETAKA YOKOE $^{2}$, KATSUHIRO UZAWA ${ }^{1}$ and HIDEKI TANZAWA ${ }^{1,2}$ \\ ${ }^{1}$ Department of Clinical Molecular Biology, Graduate School of Medicine, Chiba University, Chiba 260-8670; \\ ${ }^{2}$ Division of Dentistry and Oral Surgery, Chiba University Hospital, Chiba 260-8677, Japan
}

Received January 31, 2008; Accepted March 21, 2008

\begin{abstract}
PTEN is a tumor suppressor gene located on chromosome10q23.3. In addition to genetic mutations and deletions, the down-regulation of PTEN has been found in various malignant tumors. However, little is known about the profile of PTEN gene in oral carcinomas. In this study, the expression profiles and genetic alterations of PTEN were examined in 113 oral squamous cell carcinoma (OSCC) cases and 9 OSCC-derived cell lines. An immunohistochemical analysis showed statistically significant differences in the immunohistochemical (IHC) scores for PTEN protein in normal tissues and in cancerous regions $(\mathrm{P}=0.0104)$, suggesting that PTEN protein expression is down-regulated in OSCC. No significant correlations existed between the down-regulation of PTEN protein expression and the clinicopathological features of the tumor. Reverse transcriptionpolymerase chain reaction (RT-PCR) analysis showed a lower PTEN mRNA expression in the 9 OSCC-derived cell lines examined, as compared to the normal oral epithelium cells. However, treatment with a demethylating reagent restored PTEN mRNA expression in 4 cell lines. No genetic mutations were detected in these cell lines by polymerase chain reaction-single strand conformation polymorphism (PCR-SSCP) analysis. The results suggest that epigenetic changes may be related to the down-regulation of PTEN expression. We therefore conclude that PTEN is a crucial molecule in the tumorigenesis of OSCC.
\end{abstract}

Correspondence to: Dr Masashi Shiiba, Department of Clinical Molecular Biology, Graduate School of Medicine, Chiba University, Chiba 260-8670, Japan

E-mail: m.shiiba@faculty.chiba-u.jp

Abbreviations: OSCC, oral squamous cell carcinoma; PCR-SSCP, polymerase chain reaction-single strand conformation polymorphism

Key words: oral squamous cell carcinoma, PTEN, methylation

\section{Introduction}

The PTEN gene was recently identified as a tumor-suppressor gene located on chromosome 10q23.3 (1,2). PTEN consists of 9 exons and encodes a 403-amino acid protein with the signature motif HCXXGXXR in the protein tyrosine phosphatase active sites, and has homology with the cytoskeletal proteins, tensin and auxilin (3). Tensin is an actinbinding protein localized in focal adhesion complexes. PTEN functions as a protein phosphatase and has been implicated in the inhibition of cell migration and invasion (4), and in the inhibition of cell cycle progression (5). Moreover, PTEN exhibits phosphatase activity with lipid substrates, and it is generally believed that the major substrate of PTEN is phosphatidylinositol (3,4,5)-triphosphate (PIP-3), a product of PI3-kinase (6). The loss of PTEN function results in increased concentrations of PIP-3, leading to Akt hyperactivation. Thus, PTEN is thought to act as a tumor suppressor gene through negative regulation of the PI3-kinase/Akt cell survival pathway $(7,8)$.

Deletions or somatic mutations of PTEN have been detected in various malignant tumors, including glioma (9), breast (1), small-cell lung (10) and prostate cancer (1), endometrial carcinoma (11) and malignant melanoma (12). However, little is known about the PTEN gene in oral squamous cell carcinoma (OSCC). To clarify the role of PTEN in OSCC, 113 tissue samples of OSCC were analyzed by immunohistochemical staining, and the structural changes and methylation status of PTEN were examined in 9 cell lines derived from OSCC cells.

\section{Materials and methods}

Tissue samples and cell lines. A total of 113 pairs of tumor and corresponding normal oral mucosa samples were obtained from 113 unrelated Japanese patients with OSCC at the time of surgical resection at the Department of Oral Surgery at Chiba University Hospital. Informed consent was obtained from all patients and their families. The OSCC cases are summarized in Table I. Resected tissues were divided into two parts, one of which was frozen immediately after careful removal of the surrounding normal tissues, and was then 
Table I. Profiles of the OSCC cases examined in the study.

\begin{tabular}{|c|c|}
\hline Profile & Number \\
\hline \multirow[t]{2}{*}{ Age (years) } & Range: $21-87$ \\
\hline & Mean: 62.8 \\
\hline \multicolumn{2}{|l|}{ Gender } \\
\hline Male & 65 \\
\hline Female & 48 \\
\hline \multicolumn{2}{|c|}{ Differentiation } \\
\hline Well & 82 \\
\hline Moderate & 23 \\
\hline Poor & 8 \\
\hline \multicolumn{2}{|c|}{ Tumor size $(\mathrm{T})$} \\
\hline $\mathrm{Tl}$ & 14 \\
\hline $\mathrm{T} 2$ & 50 \\
\hline $\mathrm{T} 3$ & 17 \\
\hline $\mathrm{T} 4$ & 32 \\
\hline \multicolumn{2}{|c|}{ Regional neck metastasis (N) } \\
\hline No & 47 \\
\hline N1 & 43 \\
\hline $\mathrm{N} 2 \mathrm{a}$ & 3 \\
\hline $\mathrm{N} 2 \mathrm{~b}$ & 11 \\
\hline $\mathrm{N} 2 \mathrm{c}$ & 8 \\
\hline N3a & 1 \\
\hline \multicolumn{2}{|c|}{ Distant metastasis (M) } \\
\hline M0 & 54 \\
\hline Ml & 3 \\
\hline MX & 56 \\
\hline \multicolumn{2}{|c|}{ Stage classification } \\
\hline I & 11 \\
\hline II & 26 \\
\hline III & 21 \\
\hline IV & 55 \\
\hline
\end{tabular}

stored at $-80^{\circ} \mathrm{C}$ until DNA and RNA extraction. The other was fixed in $10 \%$ buffered formaldehyde solution for pathological diagnosis and immunohistochemical staining. A histopathological diagnosis of cancerous tissues was performed according to the International Histological Classification of Tumors by the Department of Pathology, Chiba University Hospital. The patients were diagnosed as having OSCC, and the tumor samples were examined to ensure that tumor tissue was present ( $>80 \%$ of specimens). Nine cell lines derived from human OSCC were analyzed: SAS, HSC-2, HSC-3, HSC-4, Ca9-22, Ho-1-u-1, Ho-1-N-1, SCC4 (Human Science Research Resource Bank, Osaka, Japan) and OK-92 (established in our department from tongue carcinoma).

Immunohistochemical analysis. Paraffin-embedded tissue sections $(4 \mu \mathrm{m})$ from 113 paired primary OSCCs and adjacent normal oral tissue were used for the immunohistochemical
(A) Normal epithelial tissue. IHC score: 180 .

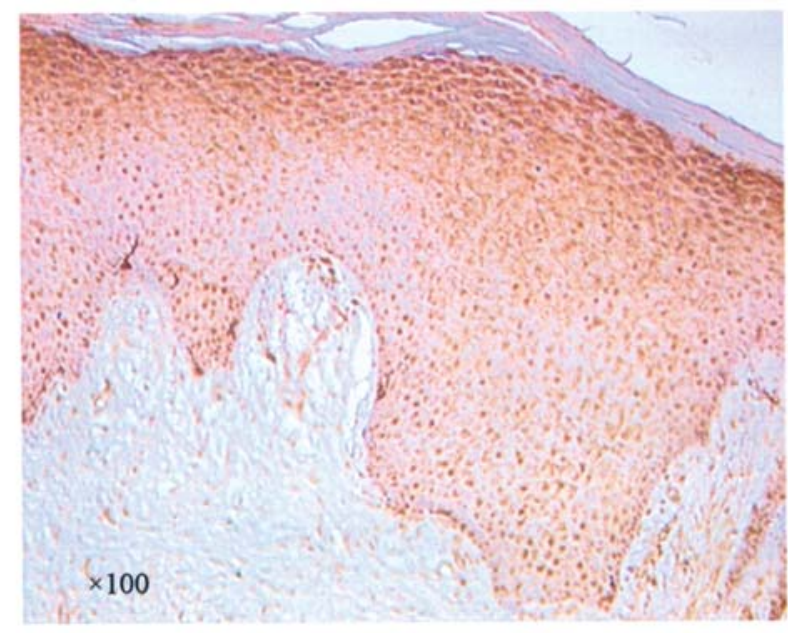

(B) OSCC. IHC score: 15 .

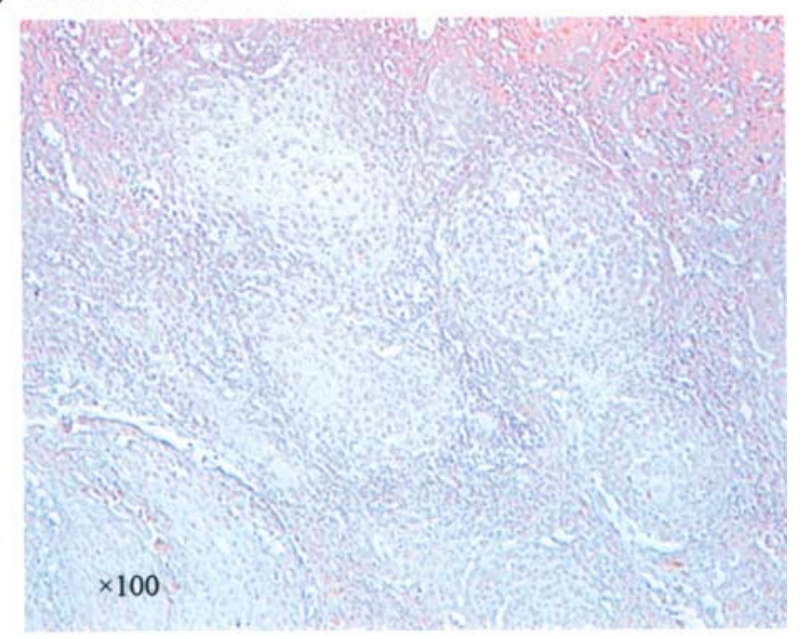

Figure 1. Immunohistochemical staining of the PTEN protein in normal and OSCC tissues. (A) Normal oral epithelial tissue. Representative image with an IHC score of 180. A strong PTEN protein expression was detected in the cytoplasm of normal oral epithelial tissue. (B) OSCC sample. Representative image showing the negative PTEN expression. Very weak immunoreactions were observed in the tumor cells. The IHC score was 15 in this case.

detection of PTEN protein. After deparaffinization and hydration, slides were heated in a microwave for $15 \mathrm{~min}$ in $0.01 \mathrm{M}$ citrate buffer ( $\mathrm{pH}$ 6.0), followed by rinsing three times in PBS solution. After quenching endogenous peroxidase activity in $0.3 \% \mathrm{H}_{2} \mathrm{O}_{2}$ for $30 \mathrm{~min}$, the sections were blocked for $2 \mathrm{~h}$ at room temperature with $5 \%$ BSA before reacting with mouse anti-human PTEN monoclonal antibody (Santa Cruz Biotechnology, Santa Cruz, CA, USA) at a dilution of 1:50. The sections were then incubated with primary antibody for $1 \mathrm{~h}$ at room temperature in a humidified chamber. The incubated sections were treated with biotinylated rabbit anti-mouse immunoglobulin (Dako Japan, Tokyo, Japan), diluted to a ratio of 1:50 in PBS for $30 \mathrm{~min}$ and incubated with biotinstreptavidin-peroxidase complex (Dako) for $30 \mathrm{~min}$, followed by color development in 3,3'-diaminobenzidine tetrahydrochloride and hydrogen peroxide mixture (Dako). The slides were then lightly counterstained with hematoxylin, dehydrated 


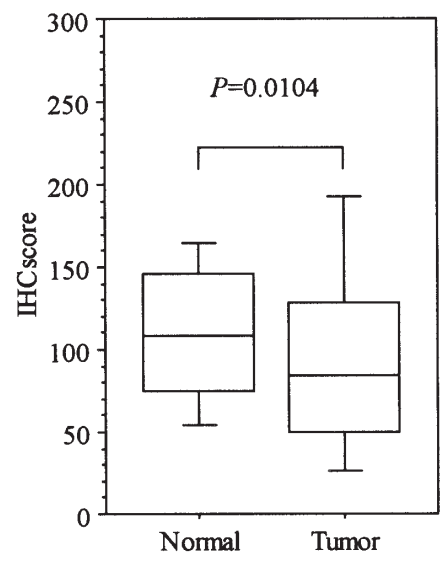

Figure 2. IHC scores in normal and tumor tissues. Median values in the normal and OSCC tissues were 109.2 and 84.0, respectively. Statistically significant differences were found between the normal and OSCC tissues $(\mathrm{P}=0.0104)$.

in a graded ethanol series, cleaned in xylene and mounted. To quantitate PTEN protein expression, a scoring method was applied (13). The mean percentage values of positive tumor cells were determined on 10 fields at $\mathrm{x} 400$ magnification in each section. The intensity of the PTEN immunoreactions was scored as follows: negative, 0 ; weak, 1 ; moderate, 2 and intense, 3 . The percentage of positive cells and the intensity of staining were multiplied to produce a PTEN immunohistochemical staining score (IHC) for each case. These judgments were made by two independent pathologists. The IHC scores were used for analyses, and the statistical significance was evaluated by Mann-Whitney's U-test.

RT-PCR analysis for PTEN mRNA expression. Total RNA was isolated from 9 OSCC-derived cell lines (OSCC, SAS, HSC-2, HSC-3, HSC-4, Ca9-22, OK-92, Ho-1-u-1, Ho-1-N-1 and SCC4) using the SV total RNA isolation system (Promega, Madison, WI, USA) according to the manufacturer's protocol. To synthesize first-strand cDNA, $1.5 \mu \mathrm{g}$ of total RNA was used for the reverse transcription reaction. The reaction was performed using a Ready-To-Go T-Primed First-Strand Kit (Amersham Pharmacia Biotech, Uppsala, Sweden). To investigate the reproducible quantitative performance of the RT-PCR assay, we titrated the amount of starting cDNA and the number of amplification cycles. All subsequent assays were carried out using parameters that yielded amplification of PTEN and GAPDH genes within a linear range. cDNAs were amplified by PCR using primers specific for the PTEN and GAPDH genes (5'-CATCTCTGCCCCCTCTGCTGA-3', sense and 5'-GGATGACCTTGCCACAGCCT-3', antisense) (Table II). cDNA preparations were performed in the presence or absence of reverse transcriptase, the latter of which acted as a control for amplification of the contaminating genomic DNA. PCR analyses were performed in a 9700 Perkin-Elmer Thermal Cycler at $95^{\circ} \mathrm{C}$ for $10 \mathrm{~min}, 30-35$ cycles of $95^{\circ} \mathrm{C}$ for $30-40 \mathrm{sec}, 55^{\circ} \mathrm{C}$ for $30 \mathrm{sec}$ and $72^{\circ} \mathrm{C}$ for $30 \mathrm{sec}$, followed by an extension step at $72^{\circ} \mathrm{C}$ for 5 min. After amplification, an aliquot of PCR products was separated on a $1.5 \%$ agarose gel, and stained with ethidium bromide. The density of the ethidium bromide-stained bands was analyzed using NIH
Image software. The results were normalized against each specific mRNA signal for $G A P D H$ within the same RNA sample. cDNA derived from normal oral epithelium was used as a positive control. Reproducibility was confirmed by processing the samples at least three times.

PCR-SSCP analysis for PTEN gene mutation. Genomic DNA was isolated from the OSCC tissue samples and cell lines, and subjected to PCR-SSCP analyses in order to examine the mutations of the PTEN gene as previously described (14). Nine sets of oligonucleotide primers were used to amplify exons 1-9 of the PTEN gene (Table II).

Evaluation of PTEN gene methylation status in OSCC-derived cell lines. The chemical 5-aza-2'-deoxycytidine (5-Aza-CdR) is known to demethylate DNA and restore transcription from promoters inactivated by hypermethylation $(15,16)$. The OSCC-derived cell lines were cultured in RPMI-1640 medium with $10 \%$ fetal bovine serum and $50 \mathrm{U} / \mathrm{ml}$ penicillin and streptomycin. Cells were treated with $2 \mu \mathrm{M}$ DNA methyltransferase inhibitor (5-Aza-CdR) for 5 days. As a control, cells were cultured at the same time without the 5-Aza-CdR reagent. The cells were subsequently washed with PBS and cultured for another 10 days without the demethylating reagent. Total RNA was extracted from the cells, and was subjected to cDNA synthesis and RT-PCR analysis in order to assess the restoration of the PTEN gene expression.

\section{Results}

Immunohistochemical analysis. An immunohistochemical analysis using anti-human PTEN monoclonal antibody was performed in order to clarify the changes in PTEN protein expression in the OSCC samples. The PTEN immunoreactions were successfully detected, primarily in the cytoplasm (Fig. 1A), and weaker positive signals were seen in the OSCC samples when compared to the enormal epithelium (Fig. 1B). The IHC scores for PTEN in normal and OSCC specimens ranged from 48 to 280 and 0 to 234 , respectively. The mean IHC scores (standard deviation) for PTEN in normal and OSCC samples were 117.2 (52.5) and 93.6 (56.7), respectively, and the median values were 109.2 and 84.0 , respectively (Fig. 2). Statistically significant differences were found between PTEN IHC scores for normal and OSCC specimens $(\mathrm{P}=0.0104)$. These results indicate that the PTEN protein expression is down-regulated in OSCC samples. However, although the relationship between the immunohistochemical data and clinicopathological features was analyzed, no significant associations were found (Table III).

Examination of the PTEN gene in OSCC cell lines. To investigate the genetic alterations in $P T E N$, a characterization of OSCC-derived cell lines was performed. First, mRNA expression in OSCC-derived cell lines was examined by RTPCR analysis. The density of the ethidium bromide-stained bands was analyzed using NIH Image software (Fig. 3A). Relative values versus the normal oral epithelium were calculated. A lower expression of PTEN mRNA was seen in the 9 cell lines examined. In HSC-3, HSC-4, Ca9-22, OK-92, Ho-1-u-1, Ho-1-N-1 and SCC4, PTEN mRNA expression was 
Table II. Specific primer sequences for amplification of the PTEN gene.

\begin{tabular}{lll}
\hline & \multicolumn{1}{c}{ Forward } & \multicolumn{1}{c}{ Reverse } \\
\hline (A) RT-PCR & 5'-ATGACAGCCATCATCAAAGAG-3' & 5'-AGGATATTGTGCAACTCTGCA-3' \\
(B) PCR-SSCP & & \\
Ex.1 & 5'-AGAGCCCCGCCACCAG-3' & 5'-AAAGAGGAGCCGCAGAA-3' \\
Ex.2 & 5'-TGACCACCTTTTATTACTCCA-3' & 5'-AGTATCTTTTTCTCGTGGCTTA-3' \\
Ex.3 & 5'-ATAGAAGGGGTATTTGTTGGA-3' & 5'-CTTCACTCTAACAAGCAGATA-3' \\
Ex.4 & 5'-TTCAGGCAATGTTTGTTA-3' & 5'-TGATAATCTGGATGACTCA-3' \\
Ex.5 & 5'-CTTATTCTGAGGTTATCTTTTTACC-3' & 5'-CTCAGAATCCAGGAAGAGGA-3' \\
Ex.6 & 5'-ACATTTTTTTTCAATTTGGATTCTC-3' & 5'-TAGATATGGTTAAGAAAACTGTTCC-3' \\
Ex.7 & 5'-ATCGTTTTTGACAGTTTG-3' & 5'-TCCCAATGAAAGTAAAGTACA-3' \\
Ex.8 & 5'-AGGACAAAATGTTTCACTTTTGGG-3' & 5'-ACATACATACAAGTCACCAACCC-3' \\
Ex.9 & 5'-CTTTCTCRTAGGTGAAGCTCTACTT-3' & 5'-TTCATGGTGTTTTATCCCTCTTGA-3' \\
\hline
\end{tabular}

(A)RT-PCR analysis for mRNA expression of PTEN gene.

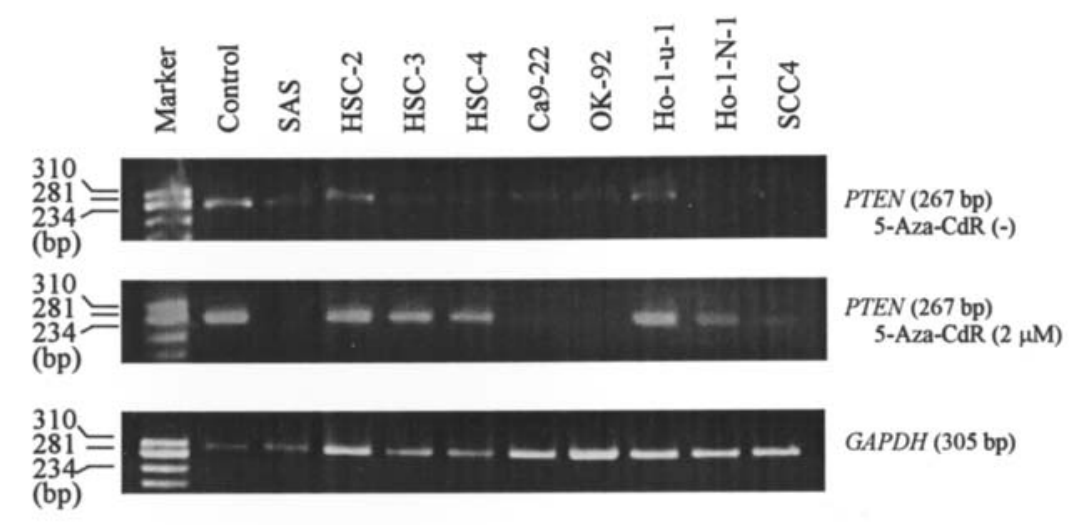

(B) Relative mRNA expression of PTEN gene against that in normal oral epithelium.

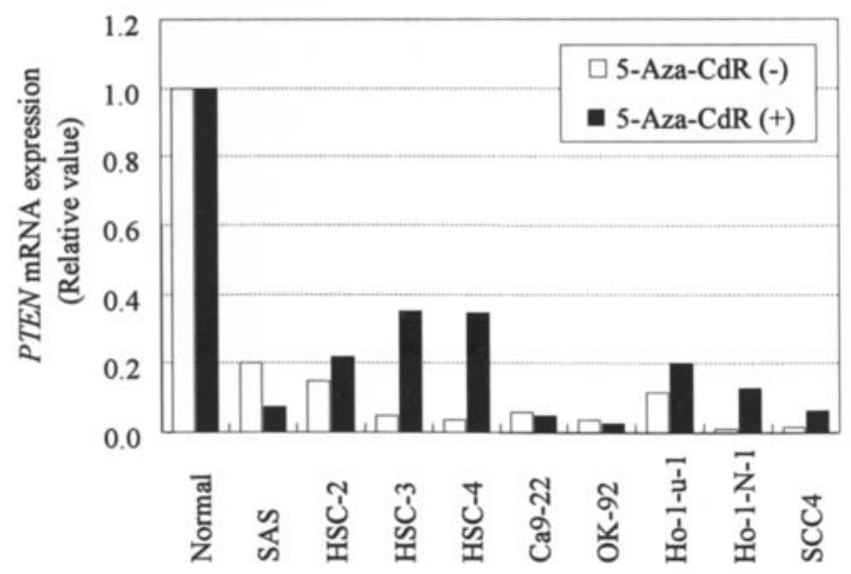

Figure 3. RT-PCR analysis for mRNA expression of the PTEN gene in OSCC-derived cell lines. (A) RT-PCR was performed with PTEN- and GAPDHspecific primers, and PCR products were subjected to electrophoresis. The panel shows the representative electrophoresis data for PCR products running on a $1.5 \%$ agarose gel. PCR products derived from normal epithelial tissue are shown as a control. (B) Band density was quantified and expressed as relative values normalized against GAPDH signals. The white columns indicate PTEN mRNA expression without the demethylating treatment. A lower expression of PTEN mRNA was observed in the 9 cell lines examined. PTEN mRNA expression of HSC-3, HSC-4, Ca9-22, OK-92, Ho-1-u-1, Ho-1-N-1 and SCC4 was below one-tenth, indicating the apparent down-regulation of PTEN mRNA. The black columns indicate PTEN mRNA expression after the demethylating treatment with 5-Aza-CdR. The graph shows the significant down-regulation of PTEN mRNA in 8 out of the 9 OSCC-derived cell lines examined. 
Table III. PTEN protein expression and clinicopathological profiles.

\begin{tabular}{|c|c|c|c|}
\hline \multirow[b]{2}{*}{ Clinicopathological profiles } & \multirow[b]{2}{*}{ Number } & IHC score & \multirow[b]{2}{*}{ P-value ${ }^{a}$} \\
\hline & & Average (SD) & \\
\hline \multicolumn{4}{|l|}{ Tumor size (T) } \\
\hline $\mathrm{T} 1+\mathrm{T} 2$ & 64 & $93.1(57.2)$ & $\mathrm{P}=0.869$ \\
\hline $\mathrm{T} 3+\mathrm{T} 4$ & 49 & $94.2(56.5)$ & \\
\hline \multicolumn{4}{|l|}{ Regional neck metastasis (N) } \\
\hline Negative & 47 & $81.2(49.4)$ & $\mathrm{P}=0.096$ \\
\hline Positive & 66 & $102.4(60.1)$ & \\
\hline \multicolumn{4}{|l|}{ Stage classification } \\
\hline $\mathrm{I}+\mathrm{II}$ & 37 & $102.7(57.1)$ & $\mathrm{P}=0.276$ \\
\hline III+IV & 76 & $89.1(56.3)$ & \\
\hline \multicolumn{4}{|l|}{ Differentiation } \\
\hline Well & 82 & $95.8(57.8)$ & $\mathrm{P}=0.373$ \\
\hline Moderate + poor & 31 & $87.6(54.0)$ & \\
\hline
\end{tabular}

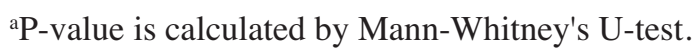

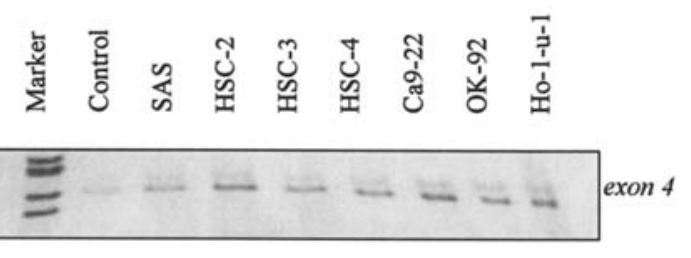

Figure 4. PCR-SSCP analysis of OSCC-derived cell lines. Representative results using PTEN exon 4-specific primers are shown. No mobility shift was observed in any of the exons (1 to 9).

below one-tenth, indicating the apparent down-regulation of PTEN mRNA (Fig. 3B). To elucidate whether the methylation status of the PTEN promoter is associated with the inactivation of PTEN, the restoration of PTEN mRNA expression by treatment with the demethylating reagent (5-Aza-CdR) was attempted. After treatment, a distinct recovery of PTEN mRNA expression was observed in HSC-3, HSC-4, Ho-1-N-1 and SCC4. These results suggest that the inactivation of PTEN may be due to hypermethylation of the PTEN gene promoter. PCR-SSCP analysis was performed to detect structural changes in the PTEN gene. No mobility shift was observed in SSCP patterns in exons 1-9 in any of the OSCC-derived cell lines examined. Representative PCR-SSCP data for exon 4 are shown in Fig. 4.

\section{Discussion}

PTEN was previously identified as a tumor suppressor gene located in human chromosome 10q23, and its association with various carcinomas has been confirmed. In the present study, PTEN protein expression in OSCC samples and PTEN mRNA expression in OSCC-derived cell lines were examined. Immunohistochemical analysis showed that PTEN protein expression was significantly lower in the OSCC regions when compared to the normal regions in OSCC samples ( $\mathrm{P}=0.0104)$, while RT-PCR analysis showed a distinctly lower expression of PTEN mRNA in 7 out of 9 OSCC-derived cell lines (77.8\%). These data suggest that the down-regulated expression of PTEN may be associated with some types of oral squamous cell carcinoma. The role of PTEN in oral carcinomas remains uncertain. Squarize et al evaluated the expression of PTEN by immunohistochemistal analysis in OSCC and concluded that aggressive tumors with a high malignancy did not express PTEN, and that PTEN is related to the histology and behavior of OSCC and may therefore be used as a prognostic marker (17). Shin et al demonstrated that the inactivation of PTEN by either mutation or loss of transcription is related to the pathogenesis of some oral cancers (18). These findings are comparable to our results. However, other investigators have suggested that no relationship between PTEN and oral carcinomas exists (19-22). Taken together, these reports suggest that PTEN plays a crucial but limited role in certain types of oral carcinoma.

Although no mutations were observed in PTEN on PCRSSCP analysis, the down-regulated expression was recovered after treatment using the demethylation reagent in 4 out of the 6 OSCC-derived cell lines that showed a lower expression of PTEN mRNA, which suggests that the down-regulation of PTEN is due to hypermethylation. Thus, we speculate that changes in epigenetic regulation are more significant than structural changes in the PTEN gene in OSCC. Similar findings were reported after an analysis of the uterine cervix SCC samples, in which no mutations and few losses of heterozygosity were observed, although PTEN promoter methylation was detected in 58\% (23). Murao et al, however, suggested that PTEN methylation was not found in cutaneous SCCs (24). Further study is necessary to determine whether this discrepancy may be based on tissue specificity. 
The physiological function of PTEN in tumors has been widely discussed, and it is generally believed that the main activity is counteracting phosphatidylinositol $(3,4,5)$-phosphate (25). The down-regulation of PTEN results in the accumulation of phosphatidylinositol $(3,4,5)$-phosphate, which leads to an increased Akt phosphorylation. Furthermore, previous studies have demonstrated that PTEN dephosphorylates focal adhesion kinase (FAK) and negatively regulates the integrin function, which is closely related to cell migration, cell growth, invasion and focal adhesion (26). We believe that similar mechanisms are involved in OSCC tumorigenesis, although no studies have been performed using an OSCC system. Pten+/- mice showed spontaneous tumor development in the intestines, lymphoid, breast, thyroid, endometrial and adrenal glands (27-30), indicating that the role of PTEN in tumorigenesis is tissue-specific. The investigation of OSCC-specific roles for PTEN inactivation is therefore important for further therapeutic approaches.

In conclusion, the present study demonstrated that the down-regulation of PTEN protein expression was found in OSCC samples, and that a significantly decreased mRNA expression of PTEN was observed in 7 out of 9 OSCC-derived cell lines $(77.8 \%)$. Although no mutational alterations were detected, the demethylation reagent-induced restoration of PTEN mRNA expression was observed in 4 out of the 7 OSCC-derived cell lines, suggesting that epigenetic changes are related to the down-regulation of PTEN expression. We thus conclude that PTEN probably plays a crucial role in the tumorigenesis of OSCC.

\section{References}

1. Li J, Yen C, Liaw D, et al: PTEN, a putative protein tyrosine phosphatase gene mutated in human brain, breast, and prostate cancer. Science 275: 1943-1947, 1997.

2. Steck PA, Pershouse MA, Jasser SA, et al: Identification of a candidate tumour suppressor gene, MMAC1, at chromosome $10 \mathrm{q} 23.3$ that is mutated in multiple advanced cancers. Nat Genet 15: 356-362, 1997.

3. Li DM and Sun H: TEP1, encoded by a candidate tumor suppressor locus, is a novel protein tyrosine phosphatase regulated by transforming growth factor beta. Cancer Res 57: 2124-2129, 1997.

4. Tamura M, Gu J, Tran H and Yamada KM: PTEN gene and integrin signaling in cancer. J Natl Cancer Inst 91: 1820-1828, 1999.

5. Hlobilkova A, Guldberg P, Thullberg M, Zeuthen J, Lukas J and Bartek J: Cell cycle arrest by the PTEN tumor suppressor is target cell specific and may require protein phosphatase activity. Exp Cell Res 256: 571-577, 2000.

6. Myers MP, Pass I, Batty IH, et al: The lipid phosphatase activity of PTEN is critical for its tumor supressor function. Proc Natl Acad Sci USA 95: 13513-13518, 1998.

7. Di Cristofano A and Pandolfi PP: The multiple roles of PTEN in tumor suppression. Cell 100: 387-390, 2000.

8. Furnari FB, Huang HJ and Cavenee WK: The phosphoinositol phosphatase activity of PTEN mediates a serum-sensitive G1 growth arrest in glioma cells. Cancer Res 58: 5002-5008, 1998.

9. Rasheed BK, Stenzel TT, McLendon RE, et al: PTEN gene mutations are seen in high-grade but not in low-grade gliomas. Cancer Res 57: 4187-4190, 1997.

10. Yokomizo A, Tindall DJ, Drabkin H, et al: PTEN/MMAC1 mutations identified in small cell, but not in non-small cell lung cancers. Oncogene 17: 475-479, 1998.

11. Sato N, Tsunoda H, Nishida M, et al: Loss of heterozygosity on 10q23.3 and mutation of the tumor suppressor gene PTEN in benign endometrial cyst of the ovary: possible sequence progression from benign endometrial cyst to endometrioid carcinoma and clear cell carcinoma of the ovary. Cancer Res 60: 7052-7056, 2000.
12. Guldberg P, thor Straten P, Birck A, Ahrenkiel V, Kirkin AF and Zeuthen J: Disruption of the MMAC1/PTEN gene by deletion or mutation is a frequent event in malignant melanoma. Cancer Res 57: 3660-3663, 1997.

13. Lombardi DP, Geradts J, Foley JF, Chiao C, Lamb PW and Barrett JC: Loss of KAI1 expression in the progression of colorectal cancer. Cancer Res 59: 5724-5731, 1999.

14. Uzawa K, Suzuki H, Tanzawa $\mathrm{H}$ and Sato K: Mutational state of p16/CDKN2 and VHL genes in squamous cell carcinoma of the oral cavity. Int J Oncol 7: 895-899, 1995.

15. Creusot F, Acs G and Christman JK: Inhibition of DNA methyltransferase and induction of Friend erythroleukemia cell differentiation by 5-azacytidine and 5-aza-2'-deoxycytidine. J Biol Chem 257: 2041-2048, 1982.

16. Timmermann S, Hinds PW and Munger K: Re-expression of endogenous p16ink4a in oral squamous cell carcinoma lines by 5-aza-2'-deoxycytidine treatment induces a senescence-like state. Oncogene 17: 3445-3453, 1998.

17. Squarize CH, Castilho RM and Santos Pinto D Jr: Immunohistochemical evidence of PTEN in oral squamous cell carcinoma and its correlation with the histological malignancy grading system. J Oral Pathol Med 31: 379-384, 2002.

18. Shin KH, Kim JM, Rho KS, Park KH, Oh JE and Min BM: Inactivation of the PTEN gene by mutation, exonic deletion, and loss of transcript in human oral squamous cell carcinomas. Int $\mathrm{J}$ Oncol 21: 997-1001, 2002.

19. Chen Q, Samaranayake LP, Zhou H and Xiao L: Homozygous deletion of the PTEN tumor-suppressor gene is not a feature in oral squamous cell carcinoma. Oral Oncol 36: 95-99, 2000.

20. Lee JI, Soria JC, Hassan KA, et al: Loss of PTEN expression as a prognostic marker for tongue cancer. Arch Otolaryngol Head Neck Surg 127: 1441-1445, 2001.

21. Mavros A, Hahn M, Wieland I, et al: Infrequent genetic alterations of the tumor suppressor gene PTEN/MMAC1 in squamous cell carcinoma of the oral cavity. J Oral Pathol Med 31: 270-276, 2002.

22. Snaddon J, Parkinson EK, Craft JA, Bartholomew C and Fulton R: Detection of functional PTEN lipid phosphatase protein and enzyme activity in squamous cell carcinomas of the head and neck, despite loss of heterozygosity at this locus. Br J Cancer 84: 1630-1634, 2001.

23. Cheung TH, Lo KW, Yim SF, et al: Epigenetic and genetic alternation of PTEN in cervical neoplasm. Gynecol Oncol 93: 621-627, 2004.

24. Murao K, Kubo Y, Ohtani N, Hara E and Arase S: Epigenetic abnormalities in cutaneous squamous cell carcinomas: frequent inactivation of the RB1/p16 and p53 pathways. Br J Dermatol 155: 999-1005, 2006.

25. Ma X, Ziel-van der Made AC, Autar B, et al: Targeted biallelic inactivation of Pten in the mouse prostate leads to prostate cancer accompanied by increased epithelial cell proliferation but not by reduced apoptosis. Cancer Res 65: 5730-5739, 2005.

26. Tamura M, Gu J, Danen EH, Takino T, Miyamoto S and Yamada KM: PTEN interactions with focal adhesion kinase and suppression of the extracellular matrix-dependent phosphatidylinositol 3-kinase/Akt cell survival pathway. J Biol Chem 274: 20693-20703, 1999.

27. Di Cristofano A, Pesce B, Cordon-Cardo C and Pandolfi PP: Pten is essential for embryonic development and tumour suppression. Nat Genet 19: 348-355, 1998.

28. Podsypanina K, Ellenson LH, Nemes A, et al: Mutation of $\mathrm{Pten} / \mathrm{Mmac1}$ in mice causes neoplasia in multiple organ systems. Proc Natl Acad Sci USA 96: 1563-1568, 1999.

29. Stambolic V, Tsao MS, Macpherson D, Suzuki A, Chapman WB and Mak TW: High incidence of breast and endometrial neoplasia resembling human Cowden syndrome in pten+/- mice. Cancer Res 60: 3605-3611, 2000

30. Suzuki A, de la Pompa JL, Stambolic V, et al: High cancer susceptibility and embryonic lethality associated with mutation of the PTEN tumor suppressor gene in mice. Curr Biol 8: 1169-1178, 1998. 